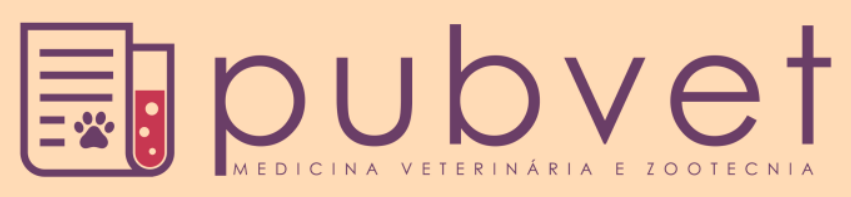

https://doi.org/10.22256/pubvet.v12n7a124.1-6

\title{
Avaliação da movimentação inter e intraestadual de aves comerciais produzidas em Santa Catarina em 2017
}

\author{
Vanessa de Medeiros Bonatelli ${ }^{1}$, Rosemberg Tartari $^{2}{ }^{\ominus}$, Priscila Belleza Maciel $^{30}$, Sara \\ Durante Felisbino $^{4}{ }^{\circ}$, Heloísa Lara Silva ${ }^{5}$, Êndrio de Elesbão Souto ${ }^{6}$, Marcos Vinicius de \\ Oliveira Neves ${ }^{7} \bullet$, Jader Nones $^{8^{*} \bullet}$ \\ ${ }^{I}$ Médica Veterinária da Companhia Integrada de Desenvolvimento Agrícola de Santa Catarina, Florianópolis-SC,Brasil, e-mail: vmbonatelli@cidasc.sc.gov.br \\ ${ }^{2}$ Médico Veterinário na Companhia Integrada de Desenvolvimento Agrícola de Santa Catarina, Florianópolis-SC, Brasil, email: rtartari@ cidasc.sc.gov.br \\ ${ }^{3}$ Médica Veterinánia da Companhia Integrada de Desenvolvimento Agrícola de Santa Catarina, Florianópolis-SC, Brasil, Doutoranda-USP, email:pbmaciel@ cidasc.Sc.gov.br \\ ${ }^{4}$ Estudante de Zootecnia - Universidade Federal de Santa Catarina (UFSC), Florianópolis-SC, Brasil, email: sarahdurante@ hotmail.com \\ ${ }^{5}$ Estudante de Zootecnia - Universidade Federal de Santa Catarina (UFSC), Florianópolis-SC, Brasil, email: heloisazoot@ gmail.com \\ ${ }^{6}$ Zootecnista-E-mail: endrioes@gmail.com \\ ${ }^{7}$ Médico Veterinário na Companhia Integrada de Desenvolvimento Agrícola de Santa Catarina, Florianópolis-SC, Brasil, email: marcosneves@ cidasc.sc.gov.br \\ ${ }^{8}$ Médico veterinário, Companhia Integrada de Desenvolvimento Agrícola de Santa Catarina, Florianópolis, Brasil, Dr. em Ciências Morfológicas - UFRJ. *Autor \\ para correspondência.E-mail:jnones@cidasc.sc.gov.br, \\ Suporte: Companhia Integrada de Desenvolvimento Agrícola de Santa Catarina (CIDASC)
}

RESUMO. A movimentação animal é um dos principais meios de disseminação de doenças. Muitas destas, pelos riscos e impactos na cadeia produtiva, fazem parte de programas de controle do Serviço de Defesa Sanitária Oficial de cada país. Com o intuito de evitar a dispersão de agentes patogênicos no Brasil, diretrizes para este controle são determinadas pelo Ministério da Agricultura, Pecuária e Abastecimento (MAPA) e executadas, em Santa Catarina, pela Companhia Integrada de Desenvolvimento Agrícola de Santa Catarina (CIDASC). O objetivo deste trabalho foi analisar e divulgar dados referentes à movimentação de aves oriundas de produções comerciais de Santa Catarina, considerando o trânsito intra e interestadual ocorrido durante o ano de 2017. Para tal, informações referentes a este período foram obtidas no banco de dados da CIDASC. Os resultados demonstraram que foram movimentados, em média por mês, no trânsito intraestadual, no período avaliado, 136.210 .943 aves, sendo 72.919 .263 para abate, 62.710.860 para engorda e 580.820 para reprodução. Os municípios catarinenses que mais movimentaram aves (origem) foram Videira, Herval d’Oeste, Faxinal dos Guedes, Chapecó e Araranguá, e os municípios que mais receberam aves (destino) foram Capinzal, Videira, Concórdia, Chapecó e Seara. Análises referentes ao trânsito interestadual demonstraram que foram movimentados mensalmente, para fora de Santa Catarina, em média, no período avaliado, 20.514.668 aves, sendo 1.641.304 para abate, 18.841.244 para engorda e 32.120 para reprodução. Os principais destinos das aves catarinenses são Paraná, Rio Grande do Sul e São Paulo. Nossos resultados demonstram a importância da avicultura na região Oeste do Estado e a ocorrência de um intenso trânsito de aves no Estado, sendo o transporte intraestadual responsável por 90,74\% desta movimentação. Devido aos riscos sanitários que o transporte de animais representa, esforços para o controle deste transporte devem ser continuamente realizados.

Palavras chaves: aves de produção, movimentação intraestadual, movimentação interestadual

\section{Evaluation of inter and intra-seasonal movement of commercial birds produced in Santa Catarina in 2017}

ABSTRACT. Animal transit is one of the main ways of disease dissemination. Because several diseases can threaten and impact the productive chain, they are targeted by control programs by the Official Health Protection Service of each country worldwide. With the 
intent of avoiding the spread of pathogenic agents in Brazil, guidelines for this control are determined by the Ministry of Agriculture, Livestock and Supply (Ministério da Agricultura, Pecuária e Abastecimento - MAPA) and applied, in Santa Catarina, by the Integrated Agricultural Development Company of Santa Catarina (Companhia Integrada de Desenvolvimento Agrícola de Santa Catarina - CIDASC). The goal of this paper is to analyze and disseminate data on the transit of commercially produced poultry from the state of Santa Catarina, while considering intrastate and interstate transit taking place in the year of 2017. For this purpose, information from this period was collected in CIDASC's database. The results show that $136,210,943$ poultry were transported every month in intrastate transit, on average, in the evaluation period: 72,919,263 for slaughter, 62,710,860 for fattening and 580,820 for breeding. The towns and cities in Santa Catarina which transported the highest number of poultry (origin) were Videira, Herval d'Oeste, Faxinal dos Guedes, Chapecó and Araranguá, and the towns and cities that received most poultry (destination) were Capinzal, Videira, Concórdia, Chapecó and Seara. Analyses of interstate transit showed that 20.514.668 poultry were transported monthly outside of Santa Catarina, on average, in the evaluated period: 1,641,304 for slaughter, 18,41,244 for fattening and 32,120 for breeding. The main destinations for the poultry from Santa Catarina are Paraná, Rio Grande do Sul and São Paulo. Our results show that poultry farming in the west of Santa Catarina is stronger and transit of poultry in the state is intense: intrastate transit accounts for $90.74 \%$ of poultry transportation. Because animal transport poses risks to health, efforts for control of this transport must be made on a regular basis.

Keywords: poultry, commercial poultry, intrastate transit, interstate transit

\section{Evaluación del movimiento inter e intra estadual de aves comerciales producidas en Santa Catarina en 2017}

RESUMEN. El desplazamiento animal es uno de los principales medios de diseminación de enfermedades. Muchas de estas, debido a los riesgos e impactos en la cadena productiva, forman parte de programas de control del Servicio de Defensa Sanitaria Oficial de cada país. Con el fin de evitar la dispersión de agentes patogénicos en Brasil, directrices para ese control son determinadas por el Ministerio de Agricultura, Ganadería y Abastecimiento (MAPA, por su sigla en portugués) y ejecutadas, en Santa Catarina, por la Compañía Integrada de Desarrollo Agrícola de Santa Catarina (CIDASC). El objetivo de este trabajo fue analizar y divulgar los datos referentes al desplazamiento de aves oriundas de producciones comerciales de Santa Catarina, considerando la circulación intra e inter estadual durante el año de 2017. Para eso, se obtuvieron las informaciones referentes a dicho período en el banco de datos de la CIDASC. Los resultados demostraron que fueron transportados, en un promedio mensual, intraestadualmente, 136.210 .943 aves, de las cuales 72.919.263 para el sacrificio, 62.710.860 para la engorda y 580.820 para la reproducción. Los municipios catarinenses que más desplazaron aves (origen) fueron Videira, Herval d`Oeste, Faxinal dos Guedes, Chapecó y Araranguá, y los municipios que más recibieron aves (destino) fueron Capinzal, Videira, Concórdia, Chapecó y Seara. Análisis relativas a la circulación interestadual demostraron un desplazamiento promedio mensual, para fuera de Santa Catarina, de 20.514.668 aves, de las cuales 1.641.304 para el sacrificio, 18.841.244 para la engorda y 32.120 para la reproducción. Los principales destinos de las aves catarinenses son Paraná, Río Grande del Sur y São Paulo. Nuestros resultados demuestran la importancia de la avicultura en la región Oeste del estado y la ocurrencia de un intenso tránsito de aves en el estado, teniendo el transporte intraestadual como responsable por el $90,74 \%$ de ese desplazamiento. Debido a los riesgos sanitarios que el transporte de animales representa, se debe realizar esfuerzos continuos para su control.

Palabras clave: aves de producción, desplazamiento intraestadual, desplazamiento interestadual 


\section{Introdução}

O trânsito pode ser considerado o principal fator facilitador da disseminação de epidemias, seja através da movimentação de animais doentes e/ou de pessoas ou fômites que tenham sido infectados (Fèvre et al., 2006).

Doenças como salmoneloses, Influenza Aviária e Doença de Newcastle, possuem alto risco de disseminação pelo trânsito. Além do trânsito de aves, o de materiais contaminados pode levar à disseminação de doenças às criações comerciais (Terregino et al., 2007; Kaleta \& Rülke, 2008; Rajan et al., 2016).

De acordo com a Síntese Anual da Agricultura de Santa Catarina (EPAGRI, 2016), o Estado possui a segunda maior produção de aves do Brasil (respondendo por $15,22 \%$ do total da produção brasileira), estando apenas atrás do Paraná $(30,60 \%)$ e à frente do Rio Grande do Sul $(13,82 \%)$. Em 2016, Santa Catarina respondeu por aproximadamente um quarto do valor exportado de carne de frango do Brasil. Além da carne de frango, Santa Catarina é líder nas exportações brasileiras de carnes de perus e patos.

Visando atender as etapas da cadeia produtiva avícola catarinense, faz-se necessário uma grande movimentação intra e interestadual de aves. Com o intuito de minimizar os riscos epidemiológicos, o Estado, por meio da CIDASC, controla o trânsito animal no território catarinense. Para tal, a CIDASC segue as diretrizes estabelecidas pelo MAPA, o qual orienta-se por meio de normas internacionais contidas no Código Sanitário para os Animais Terrestres da Organização Internacional de Saúde Animal (OIE, 2016). Deste modo, um sistema de controle de trânsito animal foi desenvolvido, o qual consiste em um serviço de defesa animal estruturado, presença de postos fixos de fiscalização sanitária nas divisas e uso de um sistema de gerenciamento agropecuário informatizado.

Este trabalho teve por objetivo traçar e divulgar um panorama da movimentação intra e interestadual de aves com origem em Santa Catarina, levando em consideração as finalidades de abate, reprodução e engorda e espécies de produção (galinha, peru, pato e marreco) transportadas em 2017.

\section{Materiais e métodos}

\section{Colheita das informações}

Os dados da movimentação animal de aves de produção comerciais foram retirados da base de dados do SIGEN+, a qual é alimentada eletronicamente, em tempo real, através do preenchimento on-line de cada GTA.

\section{Guias de trânsito animal (GTA)}

As GTAs foram emitidas no SIGEN+, conforme instruções e modelo padronizado contido na Instrução Normativa (IN) MAPA $n^{\circ} 18$, de 20 de julho de 2006 e subsequentes legislações complementares que regulamentam a GTA eletrônica (e-GTA) _ a IN MAPA n ${ }^{\circ}$ 19, 3 de maio de 2011 e a IN MAPA n ${ }^{\circ} 35$, de 02 de outubro de 2014 (Brasil, 2006; Brasil, 2011; Brasil, 2014). Tais documentos contêm informações relevantes ao Serviço de Defesa Sanitária Animal, tais como identificação da propriedade de origem e destino, finalidade, data da emissão e quantidade de animais transportados.

\section{Universo do estudo}

$\mathrm{O}$ estudo abrangeu a movimentação intra e interestadual das principais aves de produção (galinha, peru, pato e marreco) originários de Santa Catarina, sob controle do Serviço Veterinário Oficial, analisando as finalidades identificadas como principais, sendo estas: abate, engorda e reprodução.

\section{Período de obtenção dos dados}

As informações foram obtidas entre primeiro de janeiro a trinta e um de dezembro de 2017.

\section{Resultados e discussão}

Ao todo, somente em 2017, foram registrados mais de 1.200.000 GTAs emitidas em Santa Catarina, sendo que destas $427.816(35,65 \%)$ tinham relação com espécies de aves de produção. Do total destas GTAs de aves de produção, 388.217 GTAs $(90,74 \%)$ tiveram destino intraestadual e 39.599 GTAs $(9,26 \%)$ tiveram destino interestadual (igura1).

Os resultados, conforme Figura 2, demonstraram que foram movimentados, em média, por mês, no trânsito intraestadual, no período avaliado, 136.210 .943 aves, sendo 72.919.263 para abate, 62.710 .860 para engorda e 580.820 para reprodução. Considerando a movimentação intraestadual anual de animais (1.634.531.312), o mês de agosto de 2017 foi o que apresentou a maior movimentação (146.622.654) e o mês de abril a menor movimentação (125.391.476), não havendo 
variação considerada significativa entre os meses avaliados.

\section{№ de GTAs de Aves de produção por destino, 2017.}

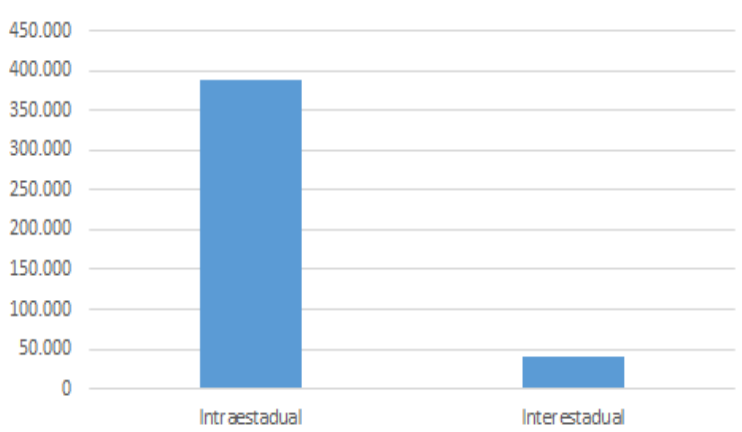

Figura 1. Número total de Guias de Transito Animal (GTAs) de aves de produção (galinha, peru, pato, marreco) por destino (intraestadual e interestadual), em 2017.

Ainda referente ao trânsito intraestadual podese observar que, considerando o somatório das principais aves de produção (galinha, peru, pato e marreco) e finalidades (abate, engorda e reprodução), os municípios catarinenses que mais movimentaram aves (origem) foram Videira, Herval d'Oeste, Faxinal dos Guedes, Chapecó e Araranguá (Quadro 1). Os municípios que mais receberam aves (destino) nessas mesmas finalidades foram Capinzal, Videira, Concórdia, Chapecó e Seara (Quadro 2).

Os municípios citados no quadro 1 corroboram com os dados apresentados na Síntese Anual da Agricultura de Santa Catarina (EPAGRI, 2016), que indica que a produção de aves em Santa Catarina encontra-se concentrada no Oeste do Estado, responsável em 2015 por $77,79 \%$ dos frangos abatidos em Santa Catarina. Com relação aos municípios indicados no quadro 2 principalmente na coluna abate, que acaba influenciando grandemente no somatório - são os locais onde estão localizados os principais abatedouros de aves do Estado, conforme o cadastro de abatedouros de aves registrados no Sigen+, base para a emissão de todas as GTAs de Santa Catarina.

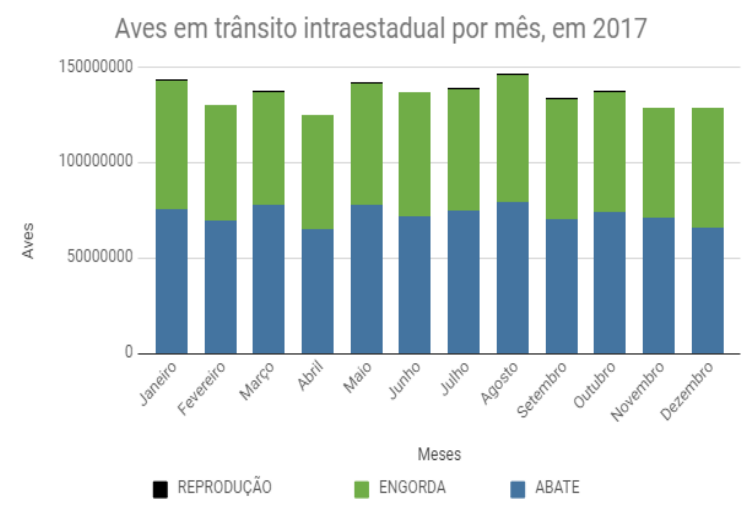

Figura 2. Número de aves em trânsito intraestadual das principais finalidades (abate, engorda, reprodução) e espécies estudadas (galinha, peru, marreco e pato), por mês, em 2017.

Análises referentes ao trânsito interestadual demonstraram que foram movimentados mensalmente, para fora de Santa Catarina, em média, no período avaliado, 20.514.668 aves, sendo 1.641.304 para abate, 18.841.244 para engorda e 32.120 para reprodução.

\begin{tabular}{|c|c|c|c|c|c|c|c|c|}
\hline $\begin{array}{l}\text { Finalidade } \\
\text { GTA }\end{array}$ & \multicolumn{2}{|c|}{ Abate } & \multicolumn{2}{|c|}{ Engorda } & \multicolumn{2}{|c|}{ Reprodução } & \multicolumn{2}{|c|}{ Somatório } \\
\hline Classificação & $\begin{array}{c}\text { Municípios } \\
\text { de origem }\end{array}$ & $\mathrm{N}^{\mathrm{o}}$ de aves & $\begin{array}{c}\text { Municípios } \\
\text { de origem }\end{array}$ & $\mathrm{N}^{\mathrm{o}}$ de aves & $\begin{array}{c}\text { Municípios } \\
\text { de origem }\end{array}$ & $\begin{array}{l}\mathrm{N}^{\circ} \text { de } \\
\text { aves }\end{array}$ & $\begin{array}{c}\text { Municípios } \\
\text { de origem }\end{array}$ & $\mathrm{N}^{\mathrm{o}}$ de aves \\
\hline 1 & Videira & 33.982 .501 & Videira & 123.982 .752 & Catanduvas & 772.807 & Videira & 157.965 .253 \\
\hline 2 & $\begin{array}{l}\text { Rio da } \\
\text { Antas }\end{array}$ & 31.547 .175 & $\begin{array}{c}\text { Faxinal dos } \\
\text { Guedes }\end{array}$ & 104.899 .008 & Capinzal & 565.298 & $\begin{array}{l}\text { Herval } \\
\text { d'Oeste }\end{array}$ & 109.544 .953 \\
\hline 3 & Ipumirim & 24.920 .954 & $\begin{array}{l}\text { Herval } \\
\text { d'Oeste }\end{array}$ & 101.847.248 & Araranguá & 552.795 & $\begin{array}{l}\text { Faxinal dos } \\
\text { Guedes }\end{array}$ & 109.190 .204 \\
\hline 4 & Seara & 24.881 .343 & Araranguá & 87.255 .097 & Orleans & 428.857 & Chapecó & 99.583 .569 \\
\hline 5 & Concórdia & 21.810 .352 & Chapecó & 83.916 .878 & Fraiburgo & 390.797 & Araranguá & 93.518 .976 \\
\hline
\end{tabular}

Quadro 1. Classificação dos principais municípios que foram origem das movimentações de aves em 2017, considerando as finalidades de abate, engorda e reprodução, assim como o somatório de todas as finalidades. 


\begin{tabular}{|l|c|c|c|c|c|c|c|c|}
\hline $\begin{array}{l}\text { Finalidade } \\
\text { GTA }\end{array}$ & \multicolumn{2}{|c|}{ Abate } & \multicolumn{2}{c|}{ Engorda } & \multicolumn{2}{c|}{ Reprodução } & \multicolumn{2}{c|}{ Somatório } \\
\hline Classificação & $\begin{array}{c}\text { Municípios } \\
\text { de destino }\end{array}$ & $\mathrm{N}^{\circ}$ de aves & $\begin{array}{c}\text { Municípios } \\
\text { de destino }\end{array}$ & $\mathrm{N}^{\circ}$ de aves & $\begin{array}{c}\text { Municípios } \\
\text { de destino }\end{array}$ & $\begin{array}{c}\mathrm{N}^{\circ} \text { de } \\
\text { aves }\end{array}$ & $\begin{array}{c}\text { Municípios } \\
\text { de destino }\end{array}$ & $\mathrm{N}^{\circ}$ de aves \\
\hline $\mathbf{1}$ & Capinzal & 119.036 .007 & Videira & 34.169 .887 & $\begin{array}{c}\text { Faxinal dos } \\
\text { Guedes }\end{array}$ & 531.894 & Capinzal & 134.689 .420 \\
\hline $\mathbf{2}$ & Videira & 100.301 .066 & $\begin{array}{c}\text { Rio das } \\
\text { Antas }\end{array}$ & 32.575 .180 & $\begin{array}{c}\text { São Miguel } \\
\text { do Oeste }\end{array}$ & 528.417 & Videira & 134.504 .094 \\
\hline $\mathbf{3}$ & Concórdia & 75.051 .501 & Seara & 22.876 .899 & $\begin{array}{c}\text { Campos } \\
\text { Novos }\end{array}$ & 422.706 & Concórdia & 89.827 .878 \\
\hline $\mathbf{5}$ & Chapecó & 66.280 .545 & Ouro & 21.793 .560 & Concórdia & 398.966 & Chapecó & 78.194 .517 \\
\hline
\end{tabular}

Quadro 2. Classificação dos principais municípios que foram destino das movimentações de aves em 2017, considerando as finalidades de abate, engorda e reprodução, assim como o somatório de todas as finalidades.

Conforme Figura 3, no trânsito interestadual, em 2017, foi registrada a maior movimentação de aves (25.139.389) em março, sendo setembro o mês com menor movimentação (17.597.204).

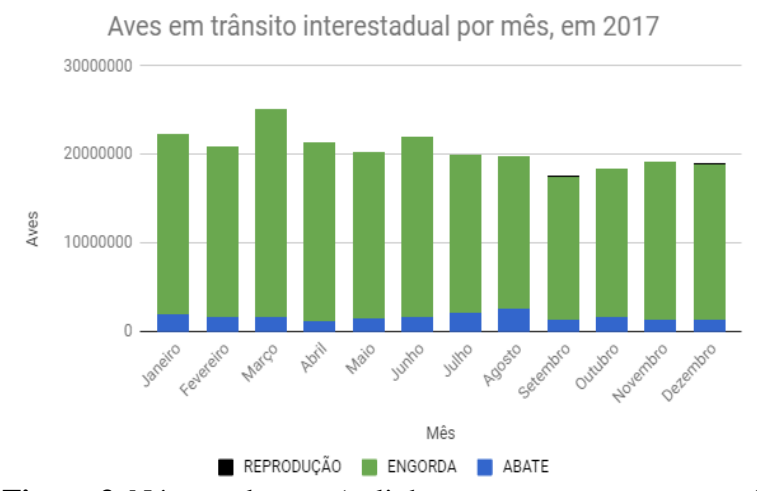

Figura 3. Número de aves (galinhas, perus, marrecos e patos) em trânsito interestadual nas principais finalidades (abate, engorda, reprodução), por mês, em 2017.

\section{Estados que mais receberam aves de produção de Santa Catarina em 2017}

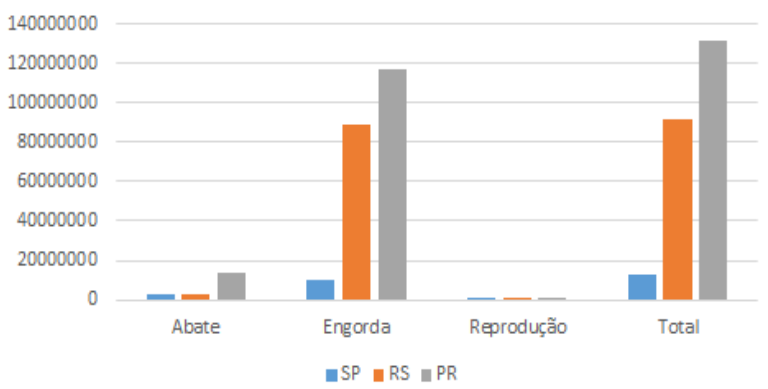

Figura 4. Estados que mais receberam aves de produção (galinhas, perus, patos e marrecos) oriundas de Santa Catarina, em 2017, por finalidade (abate, engorda e reprodução), assim como o somatório de todas as finalidades (total).
Os principais destinos dos animais enviados para fora do território catarinense foram os estados do Paraná, Rio Grande do Sul e São Paulo (Figura 4), locais que a produção avícola, assim como Santa Catarina, é bastante expressiva (ABPA, 2017).

Embora existente, dados com relação ao destino de aves de outras unidades da Federação para o território catarinense não foram, nestas análises, avaliadas.

\section{Conclusões}

Este trabalho possibilitou uma melhor compreensão sobre a movimentação intra e interestadual de aves produzidas e transportadas em Santa Catarina durante o ano de 2017. Esforços para o controle do trânsito animal devem ser continuamente realizados. Embora sejam necessários maiores estudos, os dados aqui obtidos poderão subsidiar a tomada de decisões mais assertivas para atuação de programas de controle sanitário relacionados à produção avícola e ao controle de trânsito, bem como para realização de mais aprofundados diagnósticos epidemiológicos no território catarinense.

\section{Referências}

Brasil. 2006. Instrução Normativa $\mathrm{N}^{\mathrm{o}} 18$. Estabelece o modelo da Guia de Transporte Animal (GTA). Diário Oficial da República Federativa do Brasil, Brasília, 20 de julho de 2006, p. 12. Seção 1. 
Brasil. 2011. Instrução Normativa $\mathrm{N}^{\circ} 19$. Adota o formato eletrônico da Guia de Trânsito Animal (GTA), na forma do modelo e-GTA, para movimentação, em todo o território nacional, de animais vivos, ovos férteis e outros materiais de multiplicação animal, conforme legislação vigente, cuja emissão obedecerá às diretrizes do Programa Governo Eletrônico Brasileiro. Diário Oficial da República do Federativa do Brasil, Brasília, 04 de maio de 2011, p.01. Seção 1.

Brasil. 2014. Instrução Normativa $N^{\circ} 35$. Altera o art. 1 o da Instrução Normativa no 19 , de 3 de maio de 2011. Diário Oficial da República Federativa do Brasil, Brasília, 03 de outubro de 2014, p. 3. Seção 1.

Fèvre, M. E., Bronsvoort, B. M. C., Hamilton, K. A. \& Cleaveland, S. 2006. Animal movements and the spread of infectious diseases Author links open overlay panel. Trends in Microbiology, 14, 125-131.

Kaleta, E. F. \& Rülke, C. P. A. 2008. The beginning and spread of fowl plague (H7 High Pathogenicity Avian Influenza) across Europe and Asia (1878-1955). In: Swayne, D. E. (Ed.). Avian influenza. Blackwell Publishing, Ames, Iowa, Estados Unidos.

Rajan, K., Shi, Z.\& Ricke, S.C. 2016. Current aspects of Salmonella contamination in the US poultry production chain and the potential application of risk strategies in understanding emerging hazards. Critical Reviews in Microbiology, 43, 370-392.
ABPA, Associação Brasileira de Proteína Animal. 2017. Relatório Anual. Disponível em:< http://abpa-

br.com.br/storage/files/3678c_final_abpa_rela torio_anual_2016_portugues_web_reduzido.p df>. Acesso em: 01 de março, 2018.

EPAGRI, Empresa de Pesquisa Agropecuária e Extensão Rural de Santa Catarina. 2016. Síntese Anual da Agricultura de Santa Catarina 2015-2016. Disponível em:< http://docweb.epagri.sc.gov.br/website_cepa/p ublicacoes/Sintese_2016.pdf>. Acesso em: 09 de março, 2018.

Terregino, C., De Nardi, R., Guberti, V., Scremin, M., Raffini, E., Martin, A. M., ... Capua, I. 2007.Active surveillance for avian influenza viruses in wild birds and backyard flocks in Northern Italy during 2004 to 2006. Avian Pathology, 36(4), 337-344.

OIE, World Organisation For Animal Health. Glossary. 2016 Terrestrial Animal Health Code. Disponível em:< http://www.oie.int/index.php?id=169\&L=0\&h tmfile=glossaire.htm>. Acesso em: 09 de março, 2018.

Article History:

Received 5 May 2018

Accepted 28 May 2018

Available online 28 June 2018

License information: This is an open-access article distributed under the terms of the Creative Commons Attribution License 4.0, which permits unrestricted use, distribution, and reproduction in any medium, provided the original work is properly cite. 